\title{
A Methodology Proposal of an Accessible Design for an Urban Intersection to Improve Mobility of People with Physical Disabilities
}

\author{
K Fernández ${ }^{1}$, C Santisteban², A Sánchez ${ }^{3}$ \\ ${ }^{1}$ Universidad Peruana de Ciencias Aplicadas \\ Prolongación Primavera 2390, Lima 15023, Perú \\ u201524194@upc.edu.pe; u201220175@upc.edu.pe; pcciasan@upc.edu.pe
}

\begin{abstract}
Today people with disabilities account for $15 \%$ of the world's population, making them a very high group of vulnerable people. They are exposed to a high risk of becoming potential victims of traffic accidents, and for being discriminated against when they move from place to place by physical barriers, exclusive architectural constructions, and inaccessible modes of transport. This study develops a methodology based on the User-Center Design (UCD) theoretical framework to obtain an "Inclusive Design" for an urban road intersection. The methodology prioritizes the needs of users, pedestrians, and especially those with a physical disability, into the design of the transportation infrastructure. This approach would reduce mobility barriers, allowing people to move efficiently, safely, and autonomously. In addition, this proposal incorporates universal accessibility standards and resources from the Wayfinding spatial orientation system to complement the design, making the urban environment adapt to the needs of all people without any type of distinction. The methodology is applied and validated to an existing intersection near a hospital, located in Lima. The study involves conducting surveys to people with some type of disability, before and after the proposed enhancements; thus, the effectiveness of the proposal is measured. Results indicate that applying the proposed methodology, mobility barriers are significantly eliminated by up to $95 \%$; therefore, it improves mobility, especially for people with disabilities.
\end{abstract}

Keywords: Accessible design, mobility, universal design, physical disability, motor disability, inclusive design

\section{Introduction}

At present, Universal Design is becoming more relevant worldwide due to the increase in population and the inclusion of people with disabilities in day-to-day activities. The population growth also brings an increased risk for people with disabilities to become potential victims of traffic accidents. Today around $15 \%$ of the world's population suffers from some type of disability [1], making them a very high group of vulnerable people in different areas (social, economic, cultural, among others). This vulnerability also contributes to them being exposed to latent discrimination in any aspect, which is expressed in physical barriers, exclusive architectural construction, and inaccessible modes of transport.

In Latin America the prevalence of people with disabilities has grown on a large scale, it is exposed in the current statistics of the percentage of people with some disability related to their population in countries such as Costa Rica (10.0\%), Panama (7.5\%), Brazil (23.5\%), Ecuador (6.0\%), Dominican Republic (13.5\%) and Mexico (5.0\%) [2]. The problem of mobility that exists in Latin America is associated to that most of the public spaces and transportation infrastructure had been built years or even decades ago, and they were built focuses on certain people; omitting various groups such as pregnant women, children, the elderly, and people with disabilities, whose needs to deal with the day-to-day inaccessible public spaces problem. Thus, people with disabilities have to face moving through an urban space or environment that does not suit their needs, making it difficult for pedestrians to move freely. For instance, elements such as sidewalks, bus stops, pedestrian crossings among others, which are designed in most cases in a non-accessible fashion, can causes that pedestrians choose the alternative of traveling into the road, exposing themselves to the potential risk of having an accident. The problem described above is due to the presence of mobility barriers in the streets. These barriers are the consequence of poor design infrastructure, the lack of users' information, and accessibility standards within public spaces which restrict the mobility of people, especially people with physical disabilities who travel daily through these urban environments. 
Lima - Peru is not an exception to this problem, statistics indicate that 6 out of 10 people have accessibility problems for moving around to different places in public spaces. It is documented that a large part of the population who have some type of disability have problems moving and going to different places in public spaces. Statistics indicate that $29.3 \%$ of this group of people have access problems to hospital care centers, $23 \%$ to bus stops, $18.9 \%$ to rehabilitation centers, and $18 \%$ to banking centers [3]. Despite the above mention figures, accessibility within the urban design of the city of Lima is mainly focused on prioritizing vehicles, leaving aside pedestrians. Thus, statistics indicate that $72.3 \%$ of people with motor disabilities consider that the roads through which they travel are in poor condition or have mobility barriers that hinder free transit on their route [4]. The problem mentioned above occurs in many cities in Latin America and is due to the presence of barriers in the streets due to their poor design, the lack of information in public spaces, and the lack of inclusion of accessibility standards within them that limit the mobility of people with motor disabilities who travel daily through these urban environments.

The main objective of this study is to propose a methodology based on the User Center Design (UCD) theoretical framework to place the users' needs as the main axis for the entire development of the design. It incorporates the needs of users into the design of transportation infrastructure to reduce mobility barriers and allows users to move efficiently, safely, and autonomously. The methodology is applied and validated to an existing intersection close to a hospital, located in Lima. This paper is organized into four sections. Section 2 describes the methodology used. Section 3 provides the results obtained and discusses outcomes. Section 4 contains conclusions and suggests future research directions.

\section{Methodology}

The User-Centered Design (UCD) theoretical framework is used in this study. According to the Professional Association of Information Specialists, UCD is an approach that is related to a set of methodologies and diverse techniques whose common objective is to know and understand the needs, limitations, behaviors, and characteristics of the end-user of the product, in this case, the pedestrians [5]. It should be noted that the methodological design process in the UCD is managed through a periodic process where all decisions and objectives are directed by the users, satisfying their needs. This study consists of focusing the design on the exclusive needs of people with motor disabilities. The process has four phases as is shown in figure 1. 


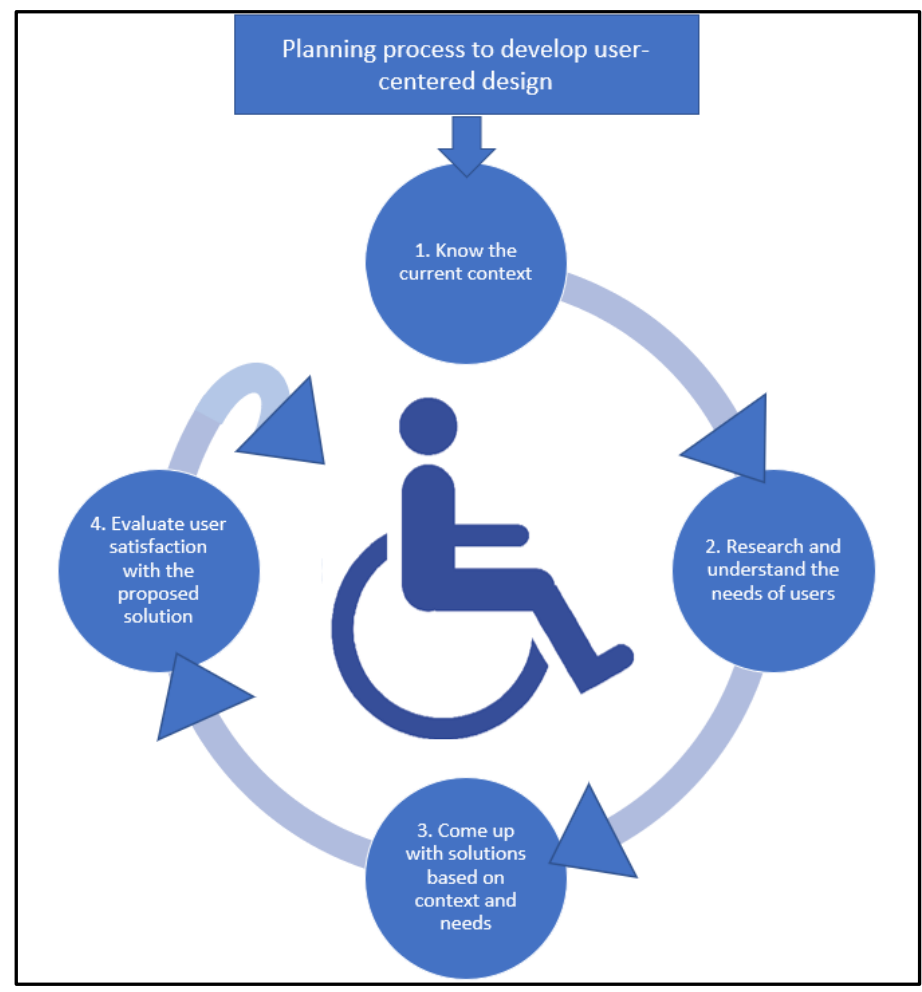

Fig. 1: Planning process for the development of accessible design focused on people with motor disabilities

As shown in figure 1, the methodology begins with the evaluation of the current situation of the selected intersection to identify the main characteristics such as geometry and the recurring problems that users constantly are exposed to.

\subsection{Identify Mobility Barriers of the Current Situation}

A prior evaluation of the current situation was conducted to identify the main design deficiencies and accessibility problems of users, to finally identify the mobility barriers at the intersection. To do this, a field investigation was conducted to collect data and process the information through the pedestrian checklist in which the characteristics and elements of the intersection are evaluated. The pedestrian checklist allows for identifying potential mobility barriers that require intervention. Table 1 displays the pedestrian checklist for the studied intersection. 
Table 1: Pedestrian checklist for the studied intersection.

\begin{tabular}{|c|c|c|c|c|}
\hline KIND & APPEARANCE & CONDITION & YES/NO & OBS \\
\hline \multirow{14}{*}{ 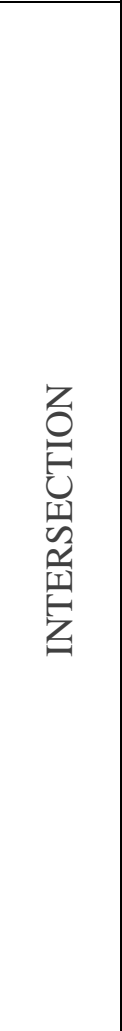 } & \multirow{7}{*}{ Geometric Design } & $\begin{array}{l}\text { The turning radio are correctly designed so that people in a } \\
\text { wheelchair can move around correctly }\end{array}$ & $\mathrm{NO}$ & \\
\hline & & $\begin{array}{l}\text { The refuge islands guaranteed adequate safety for } \\
\text { pedestrians }\end{array}$ & NO & \\
\hline & & $\begin{array}{l}\text { The condition of the pedestrian crossings is adequate, and } \\
\text { they are wide enough. }\end{array}$ & YES & \\
\hline & & $\begin{array}{l}\text { The pedestrian crossings are correctly distributed along the } \\
\text { pedestrian routes. }\end{array}$ & NO & \\
\hline & & Vehicles obstruct the crossing of pedestrians. & NO & $\begin{array}{l}\text { Only in some } \\
\text { sections }\end{array}$ \\
\hline & & $\begin{array}{l}\text { The intersections of the study area present obstacles that } \\
\text { hinder the mobility of people. }\end{array}$ & YES & \\
\hline & & There are pedestrian ramps & NO & \\
\hline & $\begin{array}{l}\text { Quality, condition, and } \\
\text { location }\end{array}$ & The condition of the pavement is adequate & NO & \\
\hline & $\begin{array}{l}\text { Continuity and } \\
\text { connectivity }\end{array}$ & $\begin{array}{l}\text { Pedestrians are properly directed to crosswalks and access } \\
\text { routes }\end{array}$ & NO & \\
\hline & \multirow{2}{*}{ Traffic characteristics } & Turning vehicles put pedestrians at risk & YES & \\
\hline & & Traffic operations create a concern for pedestrian safety & YES & \\
\hline & \multirow{3}{*}{ Traffic lights } & The intersection is signalized & YES & \\
\hline & & There are pedestrian signals at the intersection & $\mathrm{NO}$ & \\
\hline & & The pedestrian green time is sufficient for your crossing & YES & \\
\hline
\end{tabular}

Table 1 shows that most of the evaluated elements do not meet the minimum conditions. Therefore, the elements that do not meet the conditions are extracted and are considered as barriers (architectural, communication, and urban). After that, a Pareto diagram is made to find out which barriers represent a problem for people with physical disabilities. Figure 2 displays a Pareto diagram which allows for identifying the main barriers on which the proposed design should focus. This diagram was obtained from the summary of the survey carried out with users with physical disabilities.

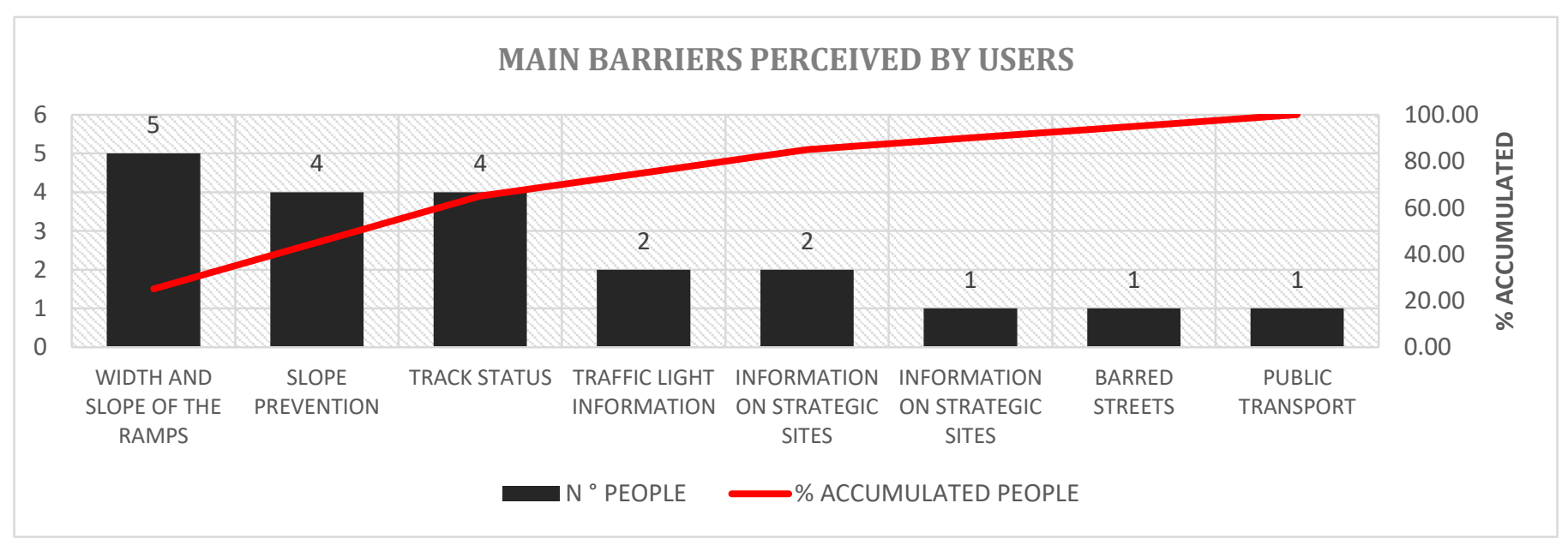

Fig. 2: Pareto diagram of the main barriers according to their degree of incidence 
On the basis of the Pareto diagram, the accessible design proposal should be focused on finding a suitable design facilitating that people with physical disabilities can move comfortably. For this reason, proposed changes in the intersection are: i) modify the width and slope of the ramps, ii) warn users in advance of the existences of ramps, and unevenness of the surface to avoid potential accidents, iii) conduct permanent maintenance of road surfaces and sidewalks, and iv) install traffic lights at locations where they can be perceived for all users. The next section explains the proposed solution for the four mobility barriers just mentioned

\subsection{Countermeasures for Mobility Barriers}

Based on the previous analysis, the countermeasures will be focused on solving:

- The geometry of the intersection. The geometry of the current situation is reviewed to identify barriers, where the proposal focuses on solving the geometric characteristics. Barriers like inadequate width and slope of the ramps were identified. The proposed design is based on the adaptation of international standards and manuals, since, in Peru, there are no accessibility standards for urban roads. The minimum width of the ramp from the crossing point to the level of the road will be $1.50 \mathrm{~m}$., so that people who use cars, walkers, or wheelchairs can move properly, as indicated in the North American ADA Standards manual. [6]. Some considerations that are taking into account before change some elements are concerning the transverse slope of the ramp, it is understood that it should not exceed $2 \%$, regardless of the design of the intersection where the ramp will be placed. If the ramps have 3 side flares, curb ramp side flares slopes should be less than $10 \%$, since they are the most suitable for efficient and problem-free circulation. Also, ramps should consider three incline flares in their design, and the maximum percentage of the slope is related to the length of the section. In this case, slopes less than $8 \%$ should be used, which allows the appropriate mobility of users reducing potential accidents.

- Elements of perception, areas with perception problems towards users were identified. Perception elements can be used to warn users of changes in slope or unevenness. The Wayfinding system resource is proposed, which is the most used in mobility systems for people with visual disabilities. This system is suitable for improving how people or users obtain information, especially applied to people with physical disabilities. This resource is known as pod tactile pavement that is made up of pieces with a continuous surface finish of buttons that will fulfill the function of warning the user of changes in slope, unevenness, changes of direction, and end of the route. In addition, as an essential requirement, it must have a contrast with respect to the surrounding pavement so it must be of a colour that guarantees this visual distinction. For example, in the study area changes in slopes across the ramps and unevenness between the curb and the pavement were identified, which cannot be noted by users. Thus, the design proposal recommends the use of the tactile pruning resource for the floors. Tactile pruning floors are strategically located before the ramps and at the edge of the sidewalk limited by the start of the pedestrian crossing. In this manner, the user in a situation of physical disability can perceive the information sent pertinently

- Maintenance work on the road surface and sidewalks. It must be permanent and not wait for them to show potholes to get to work; however, this issue only becomes important during times of political campaign. This problem is due to the fact that the cost of maintenance is foreseen within the annual budget every 5 years and in the worst cases every 10 years, depending on the alternatives of municipal expenses. On the other hand, the problem is worse when the intersection is the border between two districts, which is the case in this study. Municipalities are not clear about their responsibility; therefore, they do not pay much attention to improve the roads that separate their jurisdictions. To improve the municipal management plan, it is proposed to implement some key elements for road maintenance. First, it is important to expand the capacity of municipalities to manage maintenance with contracts of various modalities. Therefore, all companies that meet the requirements established by the entity in charge for ensuring the correct construction of public spaces can participate. Second, it was proposed to implement a stable legal framework, with agile and transparent mechanisms for adjudication and conflict resolution to accelerate the management in the process of the 
maintenance system in the district. Finally, it is recommended to set up an adequate financial and sustainable framework for the district to avoid future financial problems [7].

- Fourth and finally wisely located traffic lights. Traffic lights are urban devices found on busy roads, which have the specific objective of regulating the transit of pedestrians and vehicles. For this reason, is important that traffic lights and their time settings would be set thinking on all people, prioritizing people with disabilities. As shown in the Pareto diagram, the poor visibility of traffic lights and the inadequate width of sidewalks and crosswalks generate a constrain to the transit for people with disabilities. To face this problem, it is necessary to locate traffic lights as close as possible to the stop line of the vehicles and in this way ensure their visibility from both the sidewalk and the road. Also, traffic lights must have push buttons to activate, and they must have certain characteristics for being easily located and used by disabled people. To do this, the button must be located at a distance no greater than $1.50 \mathrm{~m}$ from the outer limit of the pedestrian crossing. Likewise, it will be located at a height that ranges from 0.90 to $1.20 \mathrm{~m}$, it must emit a sound or message indicating the confirmation for its use, it will also have a minimum diameter of $4 \mathrm{~cm}$ and it must have an icon and textual information for its respective recognition. Finally, the amber phase of the traffic lights should have a duration that at least allows a person located in the middle of the road to move to the other end of the road. In addition, the traffic light should be composed with a screen that will indicate the seconds remaining for the cycle change.

\subsection{Surveys of Users with Physical Disabilities}

A survey was conducted to understand and find out the needs of all users; thus, the design will adapt to all the user's needs, complying with all the minimum requirements established by the universal design and accessibility standards. In fact, these responses were fundamental and of great help in proposing solutions focused on the needs of people with physical disabilities. Figure 3 shows some results of the survey.

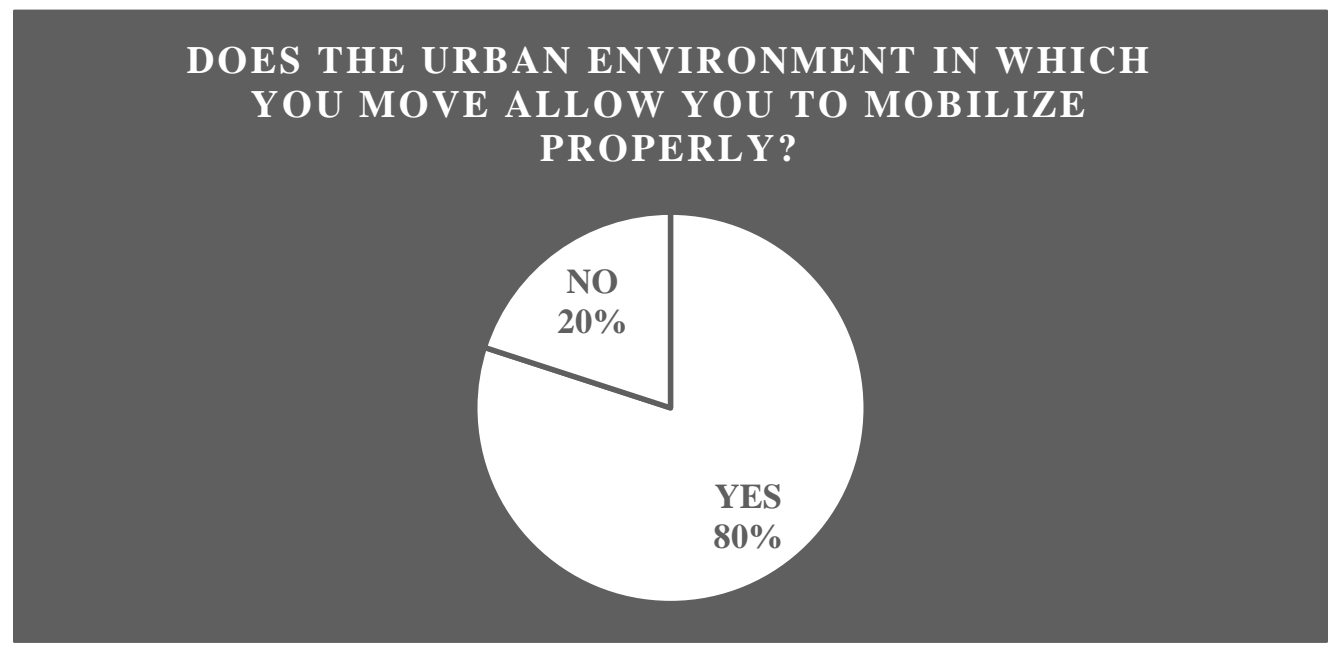

Fig. 3: Circular diagram of the percentage distribution for users who consider or not that their environment does not allow them to mobilize adequately.

Figure 3 indicates that $80 \%$ of people with disabilities do not feel that they can move properly within their environment. This is a high number of people who feel they are not considered as a part of the users in the design of the infrastructure. Table 2 shows the main barriers perceived by users. 
Table 2: Summary of the survey conducted with users on the status of the elements that make up the intersection and are considered barriers.

\begin{tabular}{|c|c|c|}
\hline BARRIERS & $\mathbf{N}^{\circ}$ PEOPLE & PERCENTAGE \\
\hline Very steep ramps & 8 & $22 \%$ \\
\hline Little perception of traffic light information & 7 & $19 \%$ \\
\hline Narrow sidewalks & 6 & $16 \%$ \\
\hline Bad condition of the tracks and sidewalks & 4 & $11 \%$ \\
\hline Very narrow spacers & 5 & $14 \%$ \\
\hline Bad bus stop design & 7 & $19 \%$ \\
\hline TOTAL & 37 & $100 \%$ \\
\hline
\end{tabular}

Table 2 indicates that inappropriate slopes of the ramps, difficulty to adequately perceive the information emitted by the traffic lights, and narrow sidewalks represent $22 \%, 19 \%$, and 16\%, respectively, from the total users. These three barriers represent more than $50 \%$ of the response of the users.

\section{Results}

Barriers within an urban road are the main indicator to classify a road as not accessible. Therefore, eliminating these barriers by at least 50\% significative improves the road infrastructure, making it more accessible, and consequently improving the mobility of users with physical disabilities. To do this, a survey was carried out on users with physical disabilities, where they evaluated the infrastructure condition and identified the mobility barriers as the first stage of this study, which was based on the seven accessibility criteria proposed by [8]. A Pugh matrix is also proposed to evaluate the elements within the study area. Results indicate that all the elements within the study area are barriers since no element has at least 4 of the suggested criteria. Finally, after making the accessible design and eliminating the barriers according to the criteria and results in the Pugh matrix, a second evaluation was conducted, following the evaluation criteria already explained. This can help us verify if the proposed design presents accessibility barriers. Table 3 shows the Pugh matrix with the scores given by the users surveyed after proposing the final design. The scoring criteria are as follow:

$1 \rightarrow$ Meets the evaluated criteria.

$0 \rightarrow$ Meets the criteria but needs improvement.

$-1 \rightarrow$ Does not meet the criteria or does not exist.

Table 3: Pugh matrix with the scores given by the surveyed users to verify the percentage of barriers removed after proposing the design.

\begin{tabular}{|c|c|c|c|c|c|c|c|c|c|}
\hline & \multicolumn{7}{|c|}{ CRITERIA } & \multirow[b]{2}{*}{ Total } \\
\hline & & $\begin{array}{c}\text { Equitable } \\
\text { use }\end{array}$ & $\begin{array}{c}\text { Flexibility in } \\
\text { use }\end{array}$ & $\begin{array}{l}\text { Simple } \\
\text { and } \\
\text { intuitive } \\
\text { use } \\
\end{array}$ & $\begin{array}{l}\text { Perceptible } \\
\text { information }\end{array}$ & $\begin{array}{l}\text { Tolerance } \\
\text { for error }\end{array}$ & $\begin{array}{c}\text { Low } \\
\text { physical } \\
\text { effort }\end{array}$ & $\begin{array}{l}\text { Size and } \\
\text { space } \\
\text { for access } \\
\text { and use }\end{array}$ & \\
\hline \multirow{3}{*}{ 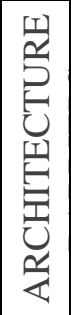 } & Ramps & 1 & 1 & 1 & 1 & 1 & 1 & 1 & 7 \\
\hline & $\begin{array}{c}\text { Central } \\
\text { separators }\end{array}$ & 1 & 1 & 1 & 1 & 1 & 1 & 1 & 7 \\
\hline & Slopes & 1 & 1 & 1 & 1 & 1 & 1 & 1 & 7 \\
\hline 0 & Zebra steps & 1 & 1 & 1 & 1 & 1 & 1 & 1 & 7 \\
\hline
\end{tabular}




\begin{tabular}{|c|c|c|c|c|c|c|c|c|c|}
\hline \multirow[t]{2}{*}{ | } & $\begin{array}{c}\text { Location of } \\
\text { lights }\end{array}$ & 1 & 1 & 1 & 1 & 0 & 1 & 1 & 6 \\
\hline & $\begin{array}{l}\text { Exclusive } \\
\text { lanes }\end{array}$ & 0 & 1 & 0 & 1 & 1 & 1 & 0 & 4 \\
\hline \multirow{2}{*}{$\begin{array}{l}Z \\
\frac{2}{\alpha} \\
\frac{\rho}{b}\end{array}$} & Whereabouts & 0 & 0 & 1 & 0 & 1 & 1 & 1 & 4 \\
\hline & $\begin{array}{c}\text { Road } \\
\text { maintenance }\end{array}$ & 1 & 1 & 1 & 1 & 1 & 1 & 1 & 7 \\
\hline
\end{tabular}

To confirm that a barrier is eliminated, it is necessary to meet at least 4 of the 7 criteria evaluated. Analyzing the Pugh matrix, after the design proposal, it is found that it was possible to eliminate the barriers by up to $100 \%$ with the application of Wayfinding system tools and adapting the design with international standards.

Also, a second user survey was conducted to find out if users consider that the proposed design would improve their mobility. Twenty users with physical disabilities were surveyed, and nineteen of them do consider that the final design improves their mobility. Figure 4 shows a pie chart with the percentage distribution for users who consider and do not consider that the proposed design would improve their mobility.

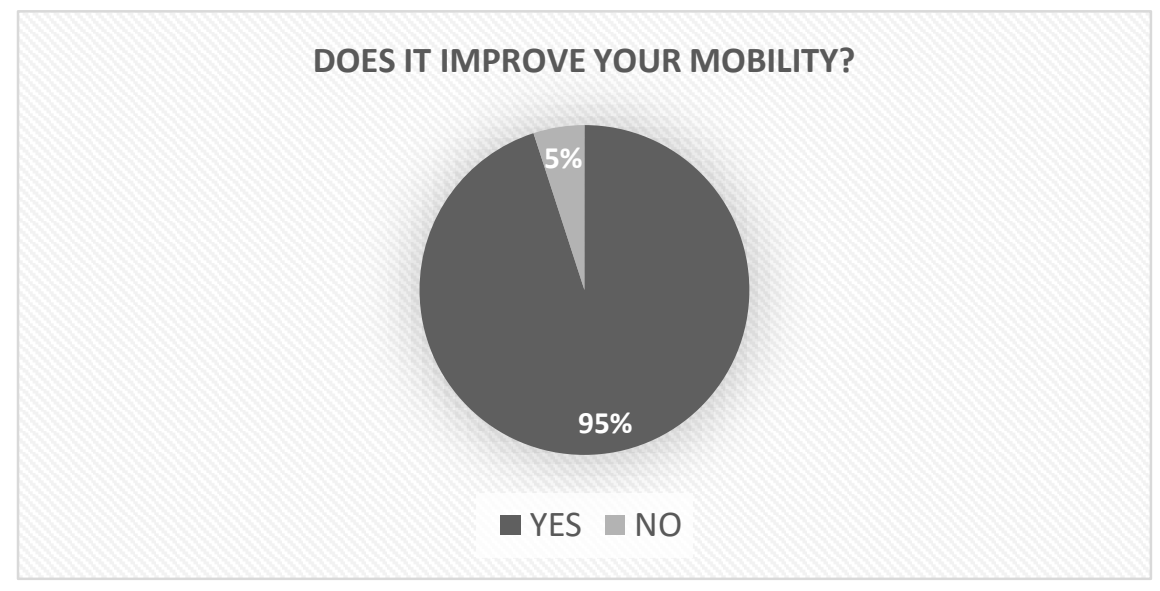

Fig. 4: Circular diagram of the percentage distribution for users who consider and do not consider that the proposed design would improve their mobility.

Finally, results indicate that $95 \%$ of those surveyed consider that the proposed design does improve their mobility and eliminate more than $50 \%$ of the existing barriers in the study area. Therefore, it allows for the conclusion that the mobility of people with physical disabilities would be improved.

\section{Conclusions}

The main reason affecting people's accessibility in society is mobility barriers that are due to the lack or the nonexistence of guidelines and manuals that consider accessible standards into the urban design infrastructure. Authorities should emphasize their public policies to enhance and convert current public spaces, which are not accessible for everyone, into an environment where people can move freely without inconvenience, especially for people with disabilities. Incorporating 
accessibility standards in the design of transportation infrastructure generates better connectivity between public spaces and a more reachable and sustainable urban environment, where any person, regardless of their physical or social condition, will be able to move effectively and safely.

Encourage the participation of people with disabilities during the design process and execution of new or existing transportation facilities such as new local roads or urban intersections helps to reduce or eliminate mobility barriers within public spaces to all users. As a result, all users, especially people with physical disabilities can move efficiently and safely across urban spaces. Incorporating geometric design changes in berms width and ramps slopes allow wheelchair users access and deal with turns without any risk of suffering an accident.

The proposed methodology promotes conducting surveys of people with physical disabilities, becoming it in a UserCentered Design, where each implemented measure is based on the user's needs. By eliminating mobility barriers, and implementing elements that provide useful information to users, the urban environment of people is transformed into a safer place, allowing them for orientation to safely arrived at their destination.

This study indicates that the observed mobility barriers directly affect the mobility of people with physical disabilities. The poor design of the ramps, existing unevenness between the berm and the pedestrian sidewalk, and surface slopes and conditions affect mobility. The importance of making a pedestrian checklist is fundamental since it allows to prioritize the barriers and propose a design that adapts to the needs of the user. Results of the survey indicate that $80 \%$ of people with disabilities cannot move adequately in public spaces, $22 \%$ consider that the ramps have an inadequate slope, making mobility difficult. The Pugh matrix indicates that with the application of the proposed methodology, barriers are significantly eliminated by up to $95 \%$, thus improving the mobility of all users and especially people with disabilities. Future studies should be focused on evaluating the effect of informality, especially in the labor market, on pedestrian mobility. It was observed that informality can also become a barrier, informal vendors among others, make it difficult for people to move or mobilize in a correct way within public spaces.

\section{References}

[1] D. Orellana, M. E. Bustos, M. Marín-Palacios, N. Cabrera-Jara and M. A. Hermida "Walk'n'roll: Mapping street-level accessibility for different mobility conditions in Cuenca, Ecuador," in Journal of Transport \& Health, 2020, vol. 16.

[2] World Health Organization (WHO). (2015). Report on the world situation of road safety.

[3] D. Cardona-Arango, A. Agudelo-Martinez, L. Restrepo-Molina and A. Segura-Cardona "The social vulnerability index regarding Medellin's disabled population," in Journal of Public Health, 2014, vol. 16, pp. 1-13.

[4] National Institute of Statistics and Informatics (INEI). (2014). Primera encuesta nacional especializada sobre discapacidad 2012.

[5] P. Chavez, "Design of an informative spatial orientation system for visually impaired people in the environment of the mass public transport Ecovía " Dept. Civil. Eng., Pontifical Catholic University of Ecuador.

[6] J. Comendador, M. E. López-Lambas and A. Monzón, "Urban Built Environment Analysis: Evidence from a Mobility Survey in Madrid. Procedia - Social and Behavioral Sciences," vol 160, pp 362-371, 2014.

[7] Y. Yang, W. Li, D. Huang, W. He, Y. Zhang and E. Merril, "An evaluation of wayfinding abilities in adolescent and young adult males with autism spectrum disorder," in Research in Autism Spectrum Disorders, 2021, vol 80.

[8] Gehl, J., and Svarre, B. (2013). How to study public life. Washington, USA: IslandPress. 